\title{
Vibrio cholerae O1 El Tor cluster in Sydney linked to imported whitebait
}

\author{
Bradley Forssman, Trish Mannes, Jennie Musto, Thomas Gottlieb, Graham Robertson, Jonathan D Natoli, \\ Craig Shadbolt, Brian Biffin and Leena Gupta
}

C holera is a rare disease in Australia; since 1991, an average of 3.4 cases of toxigenic cholera per year have been notified, most occurring in travellers returning from cholera-endemic countries. ${ }^{1}$ Cholera is caused by toxigenic Vibrio cholerae, found in aquatic environments, especially in south Asia and Africa. ${ }^{2}$ In endemic areas, water is usually the primary vehicle of cholera transmission, although secondary transmission may occur via food. ${ }^{3}$ Transmission of cholera in non-endemic areas is more commonly associated with consumption of foods, such as raw or undercooked seafood, imported from cholera-endemic regions. ${ }^{4}$ Infection can often be asymptomatic or may only cause mild diarrhoea, but severe, classic disease is characterised by vomiting and copious rice-water stool. The disease may progress to dehydration and hypovolaemic shock. ${ }^{5}$ The typical incubation period is $1-3$ days but can range from hours to 5 days. ${ }^{2,5}$

Cholera is notifiable in New South Wales under the Public Health Act 1991 (NSW). In November 2006, NSW health authorities were notified of three cases of cholera in patients with no recent travel history. Here we describe the investigation into this unusual cluster.

\section{Clinical records}

Case characteristics are summarised in the Box. Patient 1 was a 72year-old woman with fever, vomiting and severe watery diarrhoea. The vomiting resolved early in the illness. The woman presented to a hospital 6 days after onset of the watery diarrhoea and was admitted for 7 days for intravenous fluid replacement. On discharge from hospital, her symptoms had resolved.

Patient 2 was an 84-year-old woman with fever, vomiting (early in the illness), and diarrhoea. She presented to a different hospital within the same area health service as Patient 1 on the day of onset of symptoms and was admitted for 13 days for intravenous fluid replacement. The woman remained mildly unwell at discharge from hospital but made a complete recovery a week later.

Patient 3 was a 71-year-old woman also with fever, vomiting (early in the illness), and watery diarrhoea. Six days after the onset of her symptoms, she presented to the emergency department of the same hospital as Patient 2. She was diagnosed with viral gastroenteritis and was not admitted to hospital. She re-presented 2 days later with dehydration, and required intravenous fluid replacement in the emergency department. She was discharged home on the same day, and her general practitioner reported that her symptoms had resolved 5 days later.

Cholera was not initially suspected in any of these cases, as they did not fit the classic cholera picture. The cases were not notified until cholera was suspected by microbiology staff.

\section{Laboratory investigation}

Although the three patients presented to two different hospitals within the same area health service, samples from all three patients were cultured in the same laboratory. Suspicion of V. cholerae was

\section{ABSTRACT}

- Three cases of cholera in women aged 71,72 and 84 years were notified in November 2006 in Sydney, New South Wales.

- This is the first reported cluster of cholera in Australia for over 30 years, and was an unusual outbreak in patients with no history of recent travel to cholera-endemic areas.

- A food trace-back investigation found that the only exposure common to all cases was consumption of raw whitebait imported from Indonesia.

- This outbreak demonstrates that the practice of eating raw whitebait does occur in Australia, albeit in the process of taste-testing uncooked fritter batter.

- All three patients were undergoing long-term therapy with proton-pump inhibitors, which may have contributed to their susceptibility to the disease.

- A review of importation practices of food from choleraendemic regions may be required to prevent future transmission.

MJA 2007; 187: 345-347

raised when colonies with small zones of $\beta$-haemolysis were detected after culture of faeces on blood agar plates. Curved comma-shaped gram-negative bacilli were observed when colonies were Gram stained. After initial recognition on routine cultures, there was characteristic growth on thiosulfate-citrate-bile saltssucrose agar. Confirmatory serotyping indicated the culture belonged to the $\mathrm{Ol}$ serotype, subtype Ogawa. Biotyping was undertaken at the Institute of Clinical Pathology and Medical Research using the Voges-Proskauer reaction. Nucleic acid detection (by polymerase chain reaction) was used to detect genes encoding for the $V$. cholerae outer protein ompW gene ( $V$. choleraespecific) and the $V$. cholerae cholera toxin ctxA gene. This testing was done by the Microbiological Diagnostic Unit at the University of Melbourne.

\section{Public health investigation}

The Public Health Unit of the Sydney South West Area Health Service was initially notified by laboratory staff on 20 November 2006 that $V$. cholerae had been identified in faecal cultures from Patients 2 and 3. Notification of Patient l's case by laboratory staff occurred the next day. At this point, serotype and toxigenicity were not yet established. The patients were interviewed in person by public health surveillance officers to obtain symptomatology and information on possible exposures to $V$. cholerae, including travel and food history for the week before disease onset. Several interviews of all three patients were required to elicit a complete food history.

All three patients were Italian-born women aged 71-84 years who lived within a few kilometres of each other in Sydney. The 


\begin{tabular}{|lccc|}
\hline \multicolumn{4}{|c}{ Case characteristics of cholera patients, Sydney, 2006 } \\
Characteristic & Patient 1 & Patient 2 & Patient 3 \\
\hline Age (years) & 72 & 84 & 71 \\
Sex & Female & Female & Female \\
$\begin{array}{l}\text { Date and time } \\
\text { of exposure }\end{array}$ & 11 Nov 2006 & 10 Nov 2006 & 12 Nov 2006 \\
$\begin{array}{l}\text { Date and time } \\
\text { of onset }\end{array}$ & $12: 00$ & $19: 00$ & $14: 00$ \\
Incubation & Nov 2006 & 12 Nov 2006 & 15 Nov 2006 \\
period (hours) & 5 & $10: 00$ & $18: 00$ \\
Symptoms & & 39 & 76 \\
Severe watery & Yes & & \\
diarrhoea & & Yes & Yes \\
Vomiting & Yes & Yes & Yes \\
Fever & Yes & Yes & Yes \\
Hospital admission & Yes & Yes & No \\
$\begin{array}{l}\text { Duration of hospital } \\
\text { admission (days) }\end{array}$ & 7 & 13 & n/a \\
$\begin{array}{l}\text { Duration of } \\
\text { symptoms (days) }\end{array}$ & 12 & 13 & 8 \\
\hline
\end{tabular}

women did not know each other, but they all became unwell with severe watery diarrhoea within a 4-day period. None of the patients had travelled overseas in the weeks preceding onset of their symptoms, nor did they have any known contact with people who had travelled to a cholera-endemic area. All three patients were undergoing long-term therapy with proton-pump inhibitors (PPIs) for gastritis and gastro-oesophageal reflux disease.

All patients reported eating cooked seafood before illness. Further investigation revealed that the patients had independently prepared the same dish — whitebait fritters - over the same weekend. During preparation, the women would sample a spoonful of the uncooked fritter mixture of whitebait, eggs, flour and seasoning, for taste and consistency. The mixture was then cooked.

As no other food exposures were common to the patients, the raw whitebait mixture was considered to be the source of infection. Incubation periods were calculated as the time from eating the raw whitebait to symptom onset, and ranged from 5 to 76 hours. Two of the patients continued to prepare food after becoming unwell one for her large extended family and the other for her husband. However, no secondary symptomatic cases were identified among these people.

\section{Food investigation}

The whitebait was purchased from two local seafood retailers. A trace-back investigation was initiated, which involved inspection of the retail premises where the food was purchased, and tracing supplier records to determine distribution and importation of the implicated food. This investigation confirmed that both retailers had sold fresh whitebait imported from Indonesia during the patients' exposure periods. A wholesale seafood company had supplied the whitebait to both retailers, and documentation indicated that a $100 \mathrm{~kg}$ shipment of Indonesian fresh whitebait had recently arrived in Sydney, all of which was supplied to these two retailers on 31 October, and 7, 10 and 13 November 2006. None of the implicated whitebait was available for microbiological analysis at the time of enquiry on 27 November 2006.

\section{Discussion}

This cluster of locally acquired cholera in Sydney attributed to consumption of raw, imported seafood is notable because cholera is rare in Australia and is usually seen in travellers returning from cholera-endemic countries. The last reported Australian outbreak of cholera occurred in 1972, and was associated with food served on an international aircraft arriving in Australia. ${ }^{6}$ This resulted in 22 reported cases and no secondary cases. The first indigenous incident of toxigenic cholera in Australia occurred in 1977, due to exposure to contaminated drinking water, and resulted in transmission to one other person. ${ }^{7,8}$ There were five further sporadic cases of cholera that were presumed to be locally acquired over the next 10 years. $^{9}$

No secondary transmission occurred in this cluster, despite two of the patients reporting food preparation for others while unwell. It is possible that family members were exposed to low doses of $V$. cholerae, however in healthy volunteers the infectious dose has been shown to be high. ${ }^{2}$ Further, the absence of secondary transmission in this outbreak may be due to effective sanitation, good personal hygiene, and the elimination of $V$. cholerae from food by cooking.

The incubation periods in the three cases ranged from 5 to 76 hours. It is possible that the patients with shorter incubation periods consumed more whitebait during preparation of the fritter; however, the actual amount of raw whitebait consumed by the patients could not be quantified. If it is presumed that only a small amount was consumed, it is possible there was heavy contamination of the product. However, this possibility should be balanced against predisposing factors such as reduced immunocompetence associated with the ages of the women and the fact that all three patients were prescribed PPIs before illness. The main action of PPIs is to reduce gastric acid production. ${ }^{10}$ Risk of infection by gastrointestinal pathogens may be increased in people with reduced gastric acidity, ${ }^{11-14}$ and thus PPI therapy may decrease the infective dose of $V$. cholerae. ${ }^{15}$ It is possible that the patients' use of PPIs increased their susceptibility and may explain the presence of disease after ingestion of only a small amount of the raw whitebait fritter.

Indonesia was identified as the source region of the implicated whitebait. The same serotype of $V$. cholerae isolated from the patients has been identified in Indonesia. ${ }^{16}$ The Indonesian exporters certified the raw whitebait as "cholera free", however this was not verified, as the Australian Quarantine and Inspection Service (AQIS) has no formal certification arrangements with Indonesia for food safety. Unfortunately, the product was not available for retesting. It is not known whether the whitebait was kept out of refrigeration for any amount of time from the point of export to the point of consumption. Poor refrigeration may allow proliferation of the organism to an infectious dose.

Food consumed in Australia must meet the requirements of the Australia New Zealand Food Standards Code, ${ }^{17}$ which AQIS applies to foods imported into Australia. This code does not specify microbiological limits for $V$. cholerae in raw whitebait, as whitebait is intended to be consumed only after cooking, which eliminates $V$. cholerae and many other pathogens if the internal temperature reaches at least $65^{\circ} \mathrm{C} .{ }^{18}$ 
This cluster of cholera cases in Australia was associated with the consumption of raw whitebait. While the extent of this practice is unknown, this outbreak demonstrates that eating raw whitebait does occur, albeit in the process of taste-testing uncooked batter. Given the age of the patients in this outbreak, a health education intervention to prevent the eating of raw whitebait may not have been effective; however, it should not be the sole responsibility of the consumer to ensure food safety.

The World Health Organization reports on cholera outbreaks, including those resulting from consumption of contaminated imported food. There are no previously published reports of a significant outbreak of cholera resulting from commercially imported food and, for this reason, the WHO considers that the risk of acquiring cholera from imported food is low enough that an embargo on food importation from cholera-endemic areas is not necessary. ${ }^{19}$ However, the WHO does recommend that food exporters follow good hygiene practices during food handling and processing that minimise risk of potential contamination, and that importing countries such as Australia should seek confirmation that this has occurred. A review of the extent to which the WHO recommendations are followed in Australia may be required to prevent future transmission of cholera from imported food.

\section{Acknowledgements}

The authors wish to acknowledge the assistance of NSW Health and NSW Food Authority staff in this investigation, particularly Dr Jeremy McAnulty, Dr Andrew Marich, Dr Lisa Szabo, and Marianne Tegel. We also greatly appreciate the cooperation of the patients and their families.

\section{Competing interests}

None identified.

\section{Author details}

Bradley Forssman, MB BS, MPHTM, FAFPHM, Public Health Physician ${ }^{1}$ Trish Mannes, BAppSci, MPH, Epidemiologist ${ }^{1}$

Jennie Musto, MPH, Epidemiologist ${ }^{2}$

Thomas Gottlieb, MB BS, FRACP, FRCPA, Senior Specialist in Infectious

Diseases and Microbiology ${ }^{3}$

Graham Robertson, MSc, Senior Scientist ${ }^{3}$

Jonathan D Natoli, BSc, Scientific Officer ${ }^{3}$

Craig Shadbolt, PhD, Manager, Foodborne Illness Investigation Unit ${ }^{4}$

Brian Biffin, Senior Food Safety Officer ${ }^{4}$

Leena Gupta, MB BS, MPH, FAFPHM, Director ${ }^{1}$

1 Public Health Unit, Sydney South West Area Health Service, Sydney, NSW.

2 NSW Department of Health, Sydney, NSW.

3 Department of Microbiology and Infectious Diseases, Concord

Hospital, Sydney, NSW.

4 NSW Food Authority, Sydney, NSW.

Correspondence: bforssman@optusnet.com.au

\section{References}

1 Yohannes K, Roche PW, Roberts A, et al. Australia's notifiable diseases status, 2004, annual report of the National Notifiable Diseases Surveillance System. Commun Dis Intell 2006; 30: 1-79.

2 Sack DA, Sack RB, Nair GB, Siddique AK. Cholera. Lancet 2004; 363: 223 233.

3 World Health Organization. Fact sheet No. 107. Cholera. March 2000. http://www.who.int/mediacentre/factsheets/fs107/en/ (accessed Aug 2007).

4 Centers for Disease Control and Prevention (CDC). Cholera associated with food transported from El Salvador - Indiana, 1994. MMWR Morb Mortal Wkly Rep 1995; 44: 385-386.
5 Heymann DL, editor. Control of communicable diseases manual. 18th ed. Washington, DC: American Public Health Association, 2004: 103-111.

6 Sutton RG. An outbreak of cholera in Australia due to food served in flight on an international aircraft. J Hyg (Lond) 1974; 72: 441-451.

7 Rao A, Stockwell BA. The Queensland cholera incident of 1977. 1. The index case. Bull World Health Organ 1980; 58: 663-664.

8 Rogers RC, Cuffe RG, Cossins YM, et al. The Queensland cholera incident of 1977. 2. The epidemiological investigation. Bull World Health Organ 1980; 58: 665-669.

9 Bourke AT, Cossins YN, Gray BR, et al. Investigation of cholera acquired from the riverine environment in Queensland. Med J Aust 1986; 144: 229234.

10 Williams C, McColl KE. Review article: proton pump inhibitors and bacterial overgrowth. Aliment Pharmacol Ther 2006; 23: 3-10.

11 Dial S, Alrasadi K, Manoukian C, et al. Risk of Clostridium difficile diarrhea among hospital inpatients prescribed proton pump inhibitors: cohort and case-control studies. CMAJ 2004; 171: 33-38.

12 Williams C. Occurrence and significance of gastric colonization during acid-inhibitory therapy. Best Pract Res Clin Gastroenterol 2001; 15: 511 521.

13 Canani RB, Cirillo P, Roggero P, et al. Therapy with gastric acidity inhibitors increases the risk of acute gastroenteritis and communityacquired pneumonia in children. Pediatrics 2006; 117: e817-e820.

14 Neal KR, Scott HM, Slack RC, Logan RF. Omeprazole as a risk factor for campylobacter gastroenteritis: case-control study. BMJ 1996; 312: 414415.

15 Cholera and other vibrio-associated diarrhoeas. Bull World Health Organ 1980; 58: 353-374.

16 Agtini MD, Soeharno R, Lesmana M, et al. The burden of diarrhoea, shigellosis, and cholera in North Jakarta, Indonesia: findings from 24 months surveillance. BMC Infect Dis 2005; 5: 89. doi: 10.1186/1471-2334$5-89$

17 Food Standards Australia New Zealand. Food standards code and user guides. http://www.foodstandards.gov.au/thecode/ (accessed Aug 2007).

18 NSW Food Authority. Fact sheet. Correct cooking temperatures. 21 March 2005. http://www.foodauthority.nsw.gov.au/consumer/pdf/Correct\%20cooking\%20temps.pdf (accessed Aug 2007).

19 World Health Organization. Guidelines for cholera control. Geneva: WHO, 1993.

(Received 25 May 2007, accepted 14 Aug 2007)

$\square$ 